\title{
ENCUESTA SOBRE CONOCIMIENTOS Y USO DE TERAPIAS ALTERNAS A LA HORMONAL EN MUJERES MENOPÁUSICAS DE MEDELLÍN (COLOMBIA) 2009
}

\section{A survey about knowledge and use of alternative therapies to that of traditional hormone replacement in menopausal women in Medellín} (Colombia) 2009

\author{
Gloria Inés Martínez-Domínguez*, Lina María Martínez-Sánchez**, \\ María Patricia Hormaza-Ángel, M.D.***, María de los Ángeles Rodríguez-Gázquez****, \\ Gabriel Jaime Rendón-Pereira, M.D.*****, Edna Martínez-Rojas******, \\ Cristian Camilo Benítez-Restrepo****** \\ Recibido: julio 13/10 - Aceptado: enero 31/11
}

\section{RESUMEN}

Objetivo: describir el conocimiento y uso de las terapias alternativas a la hormonal en mujeres menopáusicas.

Materiales y métodos: durante 2009, se realizó un estudio descriptivo de corte transversal en mujeres menopáusicas de la ciudad de Medellín, Colombia. Los criterios de inclusión fueron edad $\geq 40$ años, haber finalizado sus ciclos menstruales al menos un año antes y no tener antecedente de histerectomía.

\footnotetext{
* Química farmacéutica, Magíster en farmacia hospitalaria. Docente, Facultad de Medicina, Universidad Pontificia Bolivariana. Medellín (Colombia). Correo electrónico: gloriai.martinez@upb.edu.co

** Bacterióloga, Especialista en Hematología. Docente de la Facultad de Medicina de la Universidad Pontificia Bolivariana. Medellín (Colombia).

*** Médica, Especialista en Ginecología y Obstetricia, subespecialista en Endocrinología. Docente, Facultad de Medicina, Universidad Pontificia Bolivariana. Medellín (Colombia).

**** Enfermera, Doctora en Salud Pública. Docente, Facultad de Medicina, Universidad Pontificia Bolivariana. Medellín (Colombia).

***** Médico residente de la Especialización en Ginecología y Obstetricia de la Facultad de Medicina de la Universidad Pontificia Bolivariana. Medellín (Colombia).

*******Estudiante de Medicina, Universidad Pontificia Bolivariana. Medellín (Colombia).
}

Las mujeres que aceptaron participar en el estudio respondieron un cuestionario con información anónima sobre variables sociodemográficas, fecha de la última menstruación, sintomatología durante la menopausia y el uso y características del consumo de terapias alternativas y de reemplazo hormonal durante la menopausia.

Resultados: un total de 274 mujeres fueron encuestadas. Un 10,6\% de las mujeres utilizaban hormonas naturales y un 46,0\% emplea productos a base de soya. Con respecto a las terapias médicas alternativas y complementarias para la sintomatología de la menopausia, diferentes a las hormonas naturales, el 32,8\% de las mujeres manifestó haber oído hablar de alguna de ellas, siendo más conocidas la homeopatía (18,6\%), el yoga $(16,8 \%)$ y la terapia de relajación (12,0\%).

Conclusión: un alto porcentaje de mujeres menopáusicas conocen y utilizan terapias alternas a la hormonal para el manejo de la sintomatología asociada a la menopausia, especialmente la de consumo de fitoestrógenos y productos a base de soya. 
Palabras clave: fitoestrógenos, menopausia, síntomas, terapias alternativas.

\section{SUMMARY}

Objective: describing knowledge about and the use of alternative therapies to traditional hormone replacement in menopausal women.

Materials and methods: a cross-sectional, descriptive study of menopausal women was carried out in the city of Medellín, Colombia, during 2009. Inclusion criteria were: being aged $\geq 40$, having finished their menstrual cycles at least one year beforehand and having no background of hysterectomy. Women who agreed to participate in the study answered a questionnaire requiring that they give anonymous information about socio-demographic variables, the date of their last menstruation, symptomatology during the menopause and the use and characteristics of alternative therapies and hormone replacement therapy during menopause.

Results: 274 women were surveyed; $10.6 \%$ of them were using natural hormones and $46.0 \%$ used soy-based products. Regarding alternative and complementary medical therapies for menopausal symptomatology different to natural hormones, $32.8 \%$ of the women stated that they had heard of some of them, the most well-known being homeopathy (18.6\%), yoga (16.8\%) and relaxation therapy (12.0\%).

Conclusion: a high percentage of menopausal women in the survey knew about and used alternative therapies to that of traditional hormone replacement for managing menopauseassociated symptomatology, especially consuming phytoestrogens and soy-based products.

Key words: phytoestrogen, menopause, symptom, alternative therapy.

\section{INTRODUCCIÓN}

Con el transcurrir de los años, se estableció que la mejor ayuda para las mujeres peri y posmenopáusicas era la Terapia de Reemplazo Hormonal (TRH) impulsada por el ginecólogo Robert Wilson en la década de los sesenta, quien propuso que el uso de estrógenos podría retrasar hasta por 20 años los síntomas menopáusicos tales como las oleadas de calor, la sudoración nocturna, la hipertensión arterial, el aumento de peso, los trastornos en el sueño y la resequedad vaginal, además de brindar protección contra la osteoporosis, la enfermedad cardiovasculary el Alzheimer. ${ }^{1,2}$ Posteriormente, se publicaron estudios clínicos que demostraron la asociación entre la TRH y el incremento de la enfermedad cardiovascular, la trombosis venosa y el cáncer de mama en mujeres posmenopáusicas, expuestas a combinaciones de estrógenos equinos y progestina. ${ }^{3}$

La Women Health Initiative (WHI) cuestionó la seguridad de la TRH y evidenció la necesidad de promover el desarrollo de terapias efectivas con mínimos riesgos para el manejo de los síntomas menopáusicos,${ }^{4}$ tales como las denominadas terapias médicas complementarias, que incluyen los fitoestrógenos u hormonas naturales, la homeopatía, la medicina ayurvédica, y la acupuntura entre otras. ${ }^{5,6}$ Diversos estudios y artículos publicados durante la última década, documentan el incremento del uso de estas terapias durante la peri y la posmenopausia, ${ }^{7-12}$ en especial el consumo de fitoestrógenos. Estos son compuestos naturales ricos en isoflavonas provenientes de semillas y frutos de algunas plantas leguminosas como soya, garbanzo, fríjol, alfalfa y trébol rojo entre otras y relacionados funcional y estructuralmente con el estradiol, la principal hormona estrogénica. ${ }^{13}$

Los resultados de WHI se difundieron no solo entre el personal médico, sino también entre las pacientes, lo que llevó a un cambio en las actitudes de las usuarias respecto a la TRH. ${ }^{14,15}$ Estas creencias pueden afectar la adherencia y efectividad de los tratamientos y plantean importantes desafíos tanto al médico y al paciente, en cuanto a si utilizar la TRH basada en estrógenos y progestágenos, utilizar las terapias con base en isoflavonas o las otras terapias alternativas antes mencionadas. ${ }^{16}$ Hay escasa información en el medio latinoamericano 
sobre las actitudes de las mujeres menopáusicas respecto a las diferentes opciones terapéuticas para el manejo de los síntomas. Por lo tanto, el presente estudio se realizó con el objetivo de determinar el conocimiento y uso de las terapias alternativas a la hormonal en mujeres menopáusicas en nuestro medio.

\section{MATERIALES Y MÉTODOS}

Se realizó un estudio descriptivo de corte transversal. Se incluyeron en el estudio las mujeres con edad igual o superior a los 40 años, que consultaron en una clínica particular y que hubieran finalizado sus ciclos menstruales al menos un año antes de la fecha de la encuesta y que no tuvieran antecedente de histerectomía, residentes en la ciudad de Medellín, Colombia en el año 2009. Medellín es la segunda ciudad más grande de Colombia, ubicada en la región central del país, con un clima promedio de 22 grados Celsius. Con una proporción esperada de consumidoras de isoflavonas de 13\%, un margen $\pm 2 \%{ }^{17}$ y una confiabilidad del $95 \%$, se estimó un tamaño muestral de 226 sujetos. Se realizó muestreo secuencial por conveniencia.

Previa firma del consentimiento informadoverbal, las mujeres que aceptaron participar en el estudio respondieron un cuestionario con información anónima sobre variables sociodemográficas, fecha de la última menstruación, presentación de sintomatología asociada a la menopausia y sobre el uso y las características del consumo de terapias alternativas y de reemplazo hormonal durante la menopausia. La información se resumió por medio de medidas de tendencia central y dispersión para variables continuas y proporciones para variables categóricas u ordinales. Para el procesamiento y análisis de la información, se utilizó el programa SPSS versión 17.0.

\section{RESULTADOS}

Durante el período de febrero a noviembre de 2009, se encuestaron 274 mujeres menopáusicas en la ciudad de Medellín. La edad promedio fue de 58,2 $\pm 8,0$ años, aproximadamente la mitad de la población encuestada $(55,1 \%)$ pertenece a estratos socioeconómicos superiores a 3, el 70,0\% tienen como mínimo de escolaridad la de algún grado de secundaria y el $97,8 \%$ se encuentra afiliado al Sistema General de Seguridad Social en Salud, bien sea al régimen contributivo o al régimen subsidiado. El promedio de años desde la última menstruación para el grupo es de 9,2 $\pm 7,5$ años. La edad promedio de presentación de la menopausia fue a los 48 años.

Los síntomas más frecuentes durante la menopausia reportados por las encuestadas fueron los vasomotores (75,5\%), la alteración en el sueño (50,4\%), los cambios de ánimo (45,3\%), el dolor de cabeza $(38,3 \%)$ y la pérdida por el interés sexual (37,5\%); es de anotar que una de cada diez mujeres no experimentó ningún síntoma. Otros síntomas mencionados con menor frecuencia fueron: la alopecia, las artralgias, la astenia, la adinamia, la flacidez muscular, la resequedad vaginal y la hiporexia.

Se reportó, además, en el grupo participante un consumo de calcio con vitamina $\mathrm{D}$ en un $43,4 \%$ y calcio sin vitamina D en $8,4 \%$; en el caso de este último, las mujeres llevan haciéndolo en promedio 35,9 $\pm 21,9$ meses.

Entre las mujeres encuestadas, el consumo actual de hormonas naturales y de productos a base de soya se estimó en 10,6\% y 46,0\%, respectivamente. Identificaron adecuadamente las hormonas naturales por sus nombres genéricos o comerciales el 94,6\% de las usuarias actuales versus el 56,6\% de las que han oído hablar de ellas. Entre los productos a base de soya, los de mayor consumo son la leche, bebidas y fórmulas nutricionales comerciales como suplementos vitamínicos, que son consumidos por el $29,9 \%$ y $11,3 \%$ del total de encuestadas, respectivamente. Del total de entrevistadas, el 23,4\% manifestó haber utilizado alguna vez en la vida la TRH (Terapia de Reemplazo Hormonal), con una frecuencia actual de uso de 16,1\%.

Entre las usuarias de TRH, el promedio de uso de esta terapia se estimó en 24,2 $\pm 15,9$ meses; al 
momento de la encuesta, una de cada tres usuarias la había suspendido principalmente por miedo a los efectos adversos (47,7\%), recomendación médica (27,2\%), no haber sentido mejoría (22,3\%), y por otras razones $(27,2 \%)$. Esta categoría incluyó aumento de peso, hemorragia uterina, la hiperprolactinemia, mastalgia, no estar cubierta por el plan de beneficios, y várices.

Con respecto a las denominadas terapias médicas alternativas y complementarias para la sintomatología de la menopausia diferente a las hormonas naturales, el 32,8\% de todas las entrevistadas manifestó haber oído hablar de alguna de ellas. Las terapias más frecuentes fueron la homeopatía, el yoga y la terapia de relajación (tabla 1).

\section{DISCUSIÓN}

La menopausia es un estado fisiológico de toda mujer cuya edad reproductiva ha culminado y que se caracteriza por el cese permanente de la menstruación después de la pérdida de la actividad ovárica. Según la literatura, la transición a la menopausia usualmente comienza a los 40 años y se instaura en promedio a los 51 años según lo reporta Randolph et al. ${ }^{18} \mathrm{La}$ edad de presentación de la menopausia fue similar a la reportada por Monterrosa et al ${ }^{19}$ en tres etnias diferentes de la población colombiana. Los síntomas se presentan en una frecuencia similar a la descrita por Gold et $a 2^{20}$ en países occidentales. Las oleadas de calor son los síntomas de mayor presentación, con 65\% a $75 \%$ y en países orientales solo son del $10 \%$ al $15 \%$. Nuestro estudio mostró un uso de vitaminas y suplementos nutricionales mayor al reportado por Bair et al (31,8\%) en población norteamericana en el estudio SWAN (Study of Women's Health Across the Nation). ${ }^{21}$ Se reportó además un consumo de calcio con y sin vitamina D similar al informado por Farrell et $a l^{22}$ para disminuir algunos riesgos asociados a la menopausia. El consumo de productos con soya en la dieta duplica lo reportado en una población italiana por Cardini et al. ${ }^{23}$

El consumo actual de la TRH en nuestro estudio es mayor con relación al estudio de Michel et al ${ }^{24}$ en población guatemalteca $(12,5 \%)$ y al de Cardini et $a l^{23}(10,5 \%)$. En cuanto al consumo previo y actual de la TRH, se observan datos similares a lo expresado por Benvenuto y Garay, ${ }^{25}$ quienes indican la tendencia a la disminución en el consumo de la TRH en mujeres que alguna vez en su vida utilizaron esta forma de terapia, por temor a sus efectos adversos, por el costo o por considerarla una terapia que interfiere con un proceso natural de las mujeres, como es la menopausia. Las terapias alternativas son menos conocidas que las reportadas en los estudios de Bair et $a 1^{21}$ y Cardini et a ${ }^{23}$, quienes muestran una incidencia y una prevalencia anual de uso de terapias médicas alternativas y complementarias del 33,5\% y $48,5 \%$ respectivamente.

\begin{tabular}{|c|c|c|}
\hline Terapia & $\begin{array}{c}\text { Ha oído hablar } \\
\text { n (\%) }\end{array}$ & $\begin{array}{c}\text { La ha utilizado } \\
\text { n (\%) }\end{array}$ \\
\hline Medicina china tradicional & $24(8,8)$ & $12(4,4)$ \\
\hline Ayurvédica & $6(2,2)$ & $1(0,4)$ \\
\hline Quiropraxia & $9(3,3)$ & $2(0,7)$ \\
\hline Yoga & $46(16,8)$ & $18(6,6)$ \\
\hline Terapia de relajación & $33(12,0)$ & $16(5,8)$ \\
\hline Homeopatía & $51(18,6)$ & $30(10,9)$ \\
\hline Aromaterapia & $18(6,6)$ & $4(1,5)$ \\
\hline Otras & $9(3,2)$ & $0(0,0)$ \\
\hline
\end{tabular}


El estudio tiene limitaciones para que los resultados sean generalizables, dado que se hizo un muestreo por conveniencia y no se indagó sobre el uso de otros medicamentos que podrían modificar la aparición de síntomas menopáusicos. Es importante, entonces, realizar estudios clínicos comparativos para obtener evidencia científica que soporte el uso de terapias diferentes a la TRH.

\section{CONCLUSIÓN}

Un importante porcentaje de mujeres menopáusicas conocen y utilizan terapias alternas a la hormonal para el manejo de la sintomatología asociada a la menopausia, especialmente la de consumo de fitoestrógenos y productos a base de soya.

\section{REFERENCIAS}

1. Nelson HD, Vesco KK, Haney E, Fu R, Nedrow A, Miller J, et al. Non hormonal therapies for menopausal hot flashes: systematic review and meta-analysis. JAMA 2006;295:2057-71.

2. Buckler H. The menopause transition: endocrine changes and clinical symptoms. J Br Menopause Soc 2005;11:61-5

3. Palmer ME, Haler C, McKinney PE, Klein-Schwartz W, Tschirgi A, Smolinske SC, et al. Adverse events associated with dietary supplements: an observational study. Lancet 2003;361:101-6.

4. Rossouw JE, Anderson GL, Prentice RL, LaCroix AZ, Kooperberg C, Stefanick ML, et al. Risks and benefits of estrogen plus progestin in healthy postmenopausal women: principal results From the Women's Health Initiative randomized controlled trial. JAMA 2002;288:321-33.

5. Geller SE, Studee L. Botanical and dietary supplements for menopausal symptoms: what works, what doesn't? J Womens Health 2005;14:634-49.

6. van der Mooren MJ, Kenemans P. Postmenopausal hormone therapy: impact on menopause-related symptoms, chronic disease and quality of life. Drugs 2004;64:821-36.

7. Dodin S, Blanchet C, Marc I. Phytooestrogènes chez la femme ménopause. Medicine/Sciences 2003;19:1030-7.

8. Bonilla CA. Isoflavonas en Ginecología, terapia no convencional. Rev Colomb Obstet Ginecol 2004;55:209-17.
9. Rees M. Alternative treatments for the menopause. Best Pract Res Clin Obstet Gynaecol 2009;23:151-61.

10. Panay R, Rees M. Alternatives to hormone replacement therapy for management of menopause symptoms. Curr Obstet Gynaecol 2005;15:259-66.

11. Warren M, Shortle B, Dominguez J. Use of alternative therapies in menopause. Best Pract Res Clin Obstet Gynaecol 2002;16:411-8.

12. Brockie J. Alternative approaches to the menopause. Rev Gynaecol Pract 2005;5:1-7.

13. Botero J, Jubiz A, Henao G. Obstetricia y Ginecología. $7^{\mathrm{a}}$ ed. Medellín: CIB; 2004. p. 597.

14. Chaikittisilpa S, Jirapinyo M, Chaovisitsaree S, Wipatavit V, Bunyaviroch S, Kanluan B, et al. Impact of women's health initiative study on attitude and acceptance of hormone replacement therapy in Thai women attending menopause clinics. J Med Assoc Thai 2007;90:628-35

15. Lukes A. Evolving issues in the clinical and managed care settings on the management of menopause following the women's health initiative. J Manag Care Pharm. 2008;14:7-13.

16. Castelo-Branco C, Peralta S, Ferrer J, Palacios S, Cornago S, Quereda F. The dilemma of menopause and hormone replacement--a challenge for women and health-care providers: knowledge of menopause and hormone therapy in Spanish menopausal women. Climacteric 2006;9:380-7

17. Wenrich TR, Cason KL. Consumption and perceptions of soy among low-income adults. J Nutr Educ Behav 2004;36:140-3.

18. Randolph JF Jr, Sowers M, Bondarenko IV, Harlow SD, Luborsky JL, Little RJ. Change in estradiol and follicle stimulating hormone across the early menopausal transition: effects of ethnicity and age. J Clin Endocrinol Metab 2004;89:1555-61.

19. Monterrosa A, Blumel JE, Chedraui P. Calidad de vida de mujeres en postmenopausia. Valoración con "Menopause Rating Scale" de tres etnias colombianas diferentes. MedUNAB 2009;12:80-5.

20. Gold EB, Sternfeld B, Kelsey JL, Brown C, Mouton C, Reame N, et al. Relation of demographic and lifestyle factors to symptoms in a multi-racial/ethnic population of women 40-55 years of age. Am J Epidemiol 2000;152:463-73.

21. Bair YA, Gold EB, Greendale GA, Sternfeld B, Adler SR, Azari R, et al. Ethnic Differences in use of complementary and alternative medicine at midlife: longitudinal results from SWAN participants. Am J Public Health 2002;92:1832-9. 
22. Farrell E. Medical choices available for management of menopause. Best Pract Res Clin Endocrinol Metab 2003;17:1-16.

23. Cardini F, Lesi G, Lombardo F, van der Sluijs C; MSCG - Menopause Survey Collaborative Group. The use of complementary and alternative medicine by women experiencing menopausal symptoms in Bologna. BMC Womens Health 2010;10:7.
24. Michel JL, Mahady GB, Veliz M, Soejarto DD, Caceres A. Symptoms, attitudes and treatment choices surrounding menopause among the Q'eqchi Maya of Livingston, Guatemala. Soc Sci Med 2006;63:732-42.

25. Benvenuto R, Garay J. Los fitoestrógenos: ¿alternativa a la TRH en climaterio? Fronteras en Obstetricia y Ginecología 2002;2:49-60.

\section{Conflicto de intereses: ninguno declarado.}

Fuente de financiación: artículo producto de la investigación que lleva el mismo nombre, financiada con recursos del Centro de Investigación para el Desarrollo y la Innovación (CIDI), y de la Facultad de Medicina de la Universidad Pontificia Bolivariana, sede Medellín. 